\title{
From pre- to postweaning: Transformation of the young calf's gastrointestinal tract ${ }^{1}$
}

\author{
Sarah J. Meale, ${ }^{\star}$ Frederique Chaucheyras-Durand,† Harma Berends, $\ddagger$ Le Luo Guan,§ and Michael A. Steele ${ }^{2}$ \\ *INRA, Unité Mixte de Recherches sur les Herbivores, UMRH1213, St Genès Champanelle, France, 63122 \\ †Lallemand Animal Nutrition, Blagnac, France, 63122 \\ †Trouw Nutrition R\&D, PO Box 220, 5830 AE, Boxmeer, the Netherlands \\ §Department of Agricultural, Food and Nutritional Science, University of Alberta, Edmonton, Canada, N1G 4T2
}

\begin{abstract}
The ruminant gastrointestinal tract (GIT) faces the challenge of protecting the host from luminal contents and pathogens, while supporting the absorption and metabolism of nutrients for growth and maintenance. The GIT of the calf in early life undergoes some of the most rapid microbial and structural changes documented in nature, and these adaptations in GIT function make the young calf susceptible to GIT diseases and disorders. Despite these challenges, the calf's GIT has a certain degree of plasticity and can sense nutrient supply and respond to bioactive ingredients. Calf GIT research has historically focused on the transition during weaning and characterizing ruminal papillae development using microscopy and digesta metabolite responses. Through the use of new molecular-based approaches, we have recently shown that delaying the age of weaning and providing a step-down weaning protocol is associated with a more gradual shift in ruminal microbiota to a postweaned state. In addition to ruminal adaptations during weaning, nutrient flow to the lower gut changes dramatically during weaning, coinciding with a wide array of structural and microbiological changes. Structural and gene expression changes suggest that the lower gut of the dairy calf undergoes alterations that may reduce barrier function when solid feeds are consumed. More recently, in vivo data revealed that the weaning transition increases total gut permeability of the calf. Interestingly, the lower gut may be able to communicate with the forestomach, meaning that a nutrient can be sensed in the lower gut and cause subsequent adaptations in the forestomach. An improved understanding of how diet, microbiota, and functional ingredients interact to affect growth and barrier func-
\end{abstract}

\footnotetext{
Received December 17, 2016.

Accepted March 17, 2017.

${ }^{1}$ Presented as part of the Non-Nutrition: The Future of Nutrition? Symposium at the Joint Annual Meeting, Salt Lake City, Utah, July 2016.

${ }^{2}$ Corresponding author: masteele@ualberta.ca
}

tion of the intestinal tract would greatly benefit the dairy calf industry. A mechanistic understanding of such adaptations would also aid in the formulation of specific management regimens and provision of functional ingredients required to characterize and enhance gut function in young calves.

Key words: forestomach, rumen, lower gut, calf

\section{INTRODUCTION}

The primary function of the gastrointestinal tract (GIT) is the digestion and absorption of nutrients. Of equal importance is the role of the GIT in protecting the host from a continually fluctuating mixture of microorganisms, toxins, and chemicals in the lumen, and to prevent unregulated translocation into portal circulation (Gäbel et al., 2002). To achieve this, the GIT continuously senses the luminal composition and adapts to support the maintenance of its integrity and enhance nutrient absorption (Furness et al., 2013). Maintaining a healthy and functional GIT is also important to animal energetics as it uses $20 \%$ of oxygen in the ruminant and accounts for $30 \%$ of metabolic and protein synthesis activities (Cant et al., 1996). Thus, the metamorphosis undergone by the GIT can affect many biological systems within the animal, making the maintenance of its function and health of great importance in dairy production systems.

With respect to GIT health in dairy production systems, digestive disorders in lactating dairy cows, such as ruminal acidosis, dominate the scientific literature. Yet, the calf is the most susceptible animal on the farm with the highest incidence of mortality and morbidity, compared with the rest of the herd. According to the most comprehensive survey available in the North American dairy industry (USDA, 2007), morbidity rates in preweaned dairy calves were estimated to be as high as $46 \%$ and mortality rates at $10 \%$, with GIT ailments being the number 1 cause. A recent survey conducted on commercial dairy farms in Ontario (Canada) and Minnesota (United States; 2,874 calves 
from 0 to $3 \mathrm{mo}$ ) reported that GIT infections were the first ailment experienced in the calf's life, with $23 \%$ of these calves receiving antibiotic treatment for diarrhea (Windeyer et al., 2014). Along with a high degree of infection, antibiotic treatments, and stress during the preweaning phase, weaning represents one of the most dramatic GIT transformations in nature and is associated with distress, depressed growth, and impaired GIT health (Khan et al., 2011, 2016). Consequently, the period from birth to weaning is a time of extreme GIT challenges, yet the short- and long-term biological outcomes of altered GIT development are still poorly understood.

Physiological development of the GIT has been characterized using dissection techniques and the analysis of digesta and blood metabolite responses in calves for some time. Yet, the advancement of different fields of science, such as high-throughput sequencing, have effectively opened the door to new capabilities of characterizing GIT compartments under specific nutritional programs or challenges. In addition to the evolution of scientific techniques, the dairy industry has also undergone a change in feeding regimen, from feeding restricted levels of milk that limit growth rates preweaning to feeding elevated levels of milk that are close to ad libitum or natural intakes and yield greater growth rates preweaning. This change undoubtedly affects GIT physiology, molecular biology, and microbiology; however, there remains a paucity of information in this area that limits further enhancements in calf biology and management.

Therefore, this review aims to summarize our current knowledge of the transformation the calf GIT undergoes during the pre- and postweaning periods. We begin with the forestomach, which has received most of the attention in dairy calf research, followed by the lower gut. The biological relevance of new molecular-based research on calf performance, GIT health and function, and overall animal welfare are discussed, and the largest knowledge gaps around GIT biology are pinpointed. Furthermore, we attempt to elucidate potential control mechanisms of gut function by reviewing microbialhost and host-host signaling (Figure 1).

\section{FORESTOMACH ADAPTATIONS}

In the newborn calf, dietary requirements are fulfilled by the uptake of colostrum, which is digested in the abomasum and subsequent GIT to provide energy, essential nutrients, as well as immunity molecules. Continuing through the preweaning phase the majority of energy intake originates from milk or milk replacer (MR), which due to reflexive closure of the reticular groove, bypasses the rumen and directly enters the abomasum (Baldwin et al., 2004). The role of the abomasum is often overshadowed by the rumen, yet many digestive disorders and ailments during the preweaning phase are related to impaired abomasal function. For example, there is concern regarding the development of depressed abomasal pH (Ahmed et al., 2002), ulceration (Ahmed et al., 2002; Marshall, 2009) and overflow of milk into the rumen (termed ruminal drinking; Berends, 2014) and reduced insulin sensitivity (Bach et al., 2013) in calves fed an elevated level of milk with a low meal frequency ( $<2$ meals per day). Several recent studies, however, have challenged our understanding of abomasal capacity in young calves (Ellingsen et al., 2016) and by showing a limited effect of larger meals (4 L, 2 times per day) on hyperglycemia (MacPherson et al., 2016). In the second experiment, calves adapted to larger meal sizes by reducing their abomasal emptying rate (MacPherson et al., 2016), a process that has been linked to the control of pancreatic insulin secretion (Stahel et al., 2016), suggesting that nutrient sensing likely occurs in lower gut neuroendocrine cells which transmit feedback that affects functionality of the forestomach, namely the abomasum.

Most research on the transformation of the calf GIT focuses on rumen maturation, which is believed to be initiated by the consumption of solid feed, but arguably starts much earlier, with solid food intake fueling a rapid increase in ruminal fermentation. The production and absorption of resulting fermentation end products (i.e., VFA) stimulate ruminal papillae development (Sander et al., 1959; Baldwin et al., 2004; Suárez et al., 2006), whereas the physical structure of substrates such as roughages contribute to muscular development and expansion of ruminal volume (Tamate et al., 1962; Stobo et al., 1966), and stimulate rumination and saliva flow to the rumen (Hodgson, 1971). The main enzymatic activities (fibrolysis, amylolysis, proteolysis, and ureolysis) of ruminal microbiota have been observed in the rumen from 4 (Sahoo et al., 2005) or 10 (Kmet et al., 1986) d of age. In excess of 60 glycoside hydrolase microbial genes are present in the rumen at early stages of life, suggesting great potential for plant carbohydrate metabolism even in the absence of regular plant cell wall intake ( $\mathrm{Li}$ et al., 2012), which is confirmed by the measurement of fibrolytic activities in the young calf rumen (Rey et al., 2012; Jiao et al., 2015). Rey et al. (2012) measured both ruminal enzyme activities and fermentative capacity in dairy calves from birth through weaning. In the first month after birth, high levels of enzymatic activities were measured in addition to changes in ruminal $\mathrm{pH}$, which decreased in a time pattern contrary to that of total VFA concentration. At $12 \mathrm{~d}$ of age, total VFA concentration plateaued (84.4 $\mathrm{m} M$ ), in agreement with earlier findings (Anderson et 
al., 1987; Beharka et al., 1991). From that point onward, the intake of solid feed strongly increased, despite a fairly stable ruminal volume and ruminal proteolytic and ureolytic enzymatic activity (Rey et al., 2012). Whether the latter factors are a determinant of feed intake remains unclear.

The process of weaning, where milk is completely removed from the diet, results in significant morphological changes, as the total capacity of the rumen increases from 30 to $70 \%$ of the entire GIT (Warner et al., 1965), with the length and surface area of papillae also in- creasing (Yonekura et al., 2002). Additionally, as a calf ages, the ketogenic capacity of the rumen must develop to that of a mature rumen, as 60 to $80 \%$ of all VFAs are absorbed across the rumen wall, with 75 to $90 \%$ of absorbed butyrate being metabolized by the rumen epithelium (Allen, 1997). However, evidence suggests the rumen may not in fact be ready to assume this primary role of nutrient absorption and metabolism before $8 \mathrm{wk}$ of age. A recent study by Eckert et al. (2015) observed declining serum BHB levels in calves during the stepdown week of a weaning protocol from a high plane of

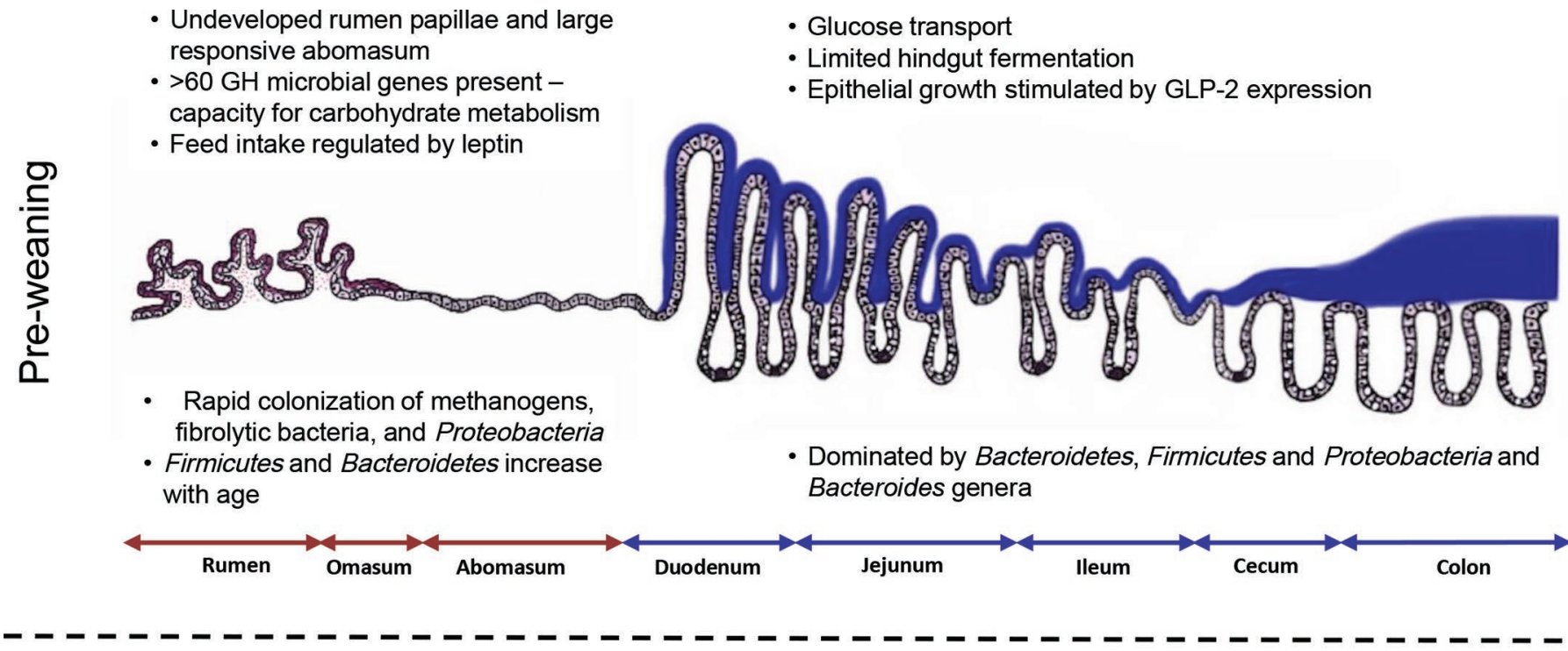

- Rapid increase in growth \& absorption

- TGF $\beta 1$ upregulated
- Decreased glucose transport \& increased hindgut fermentation

- Stimulation of host pattern recognition receptors

- Increased permeability

- Decreased glucose transport

- Downregulation of TLRs
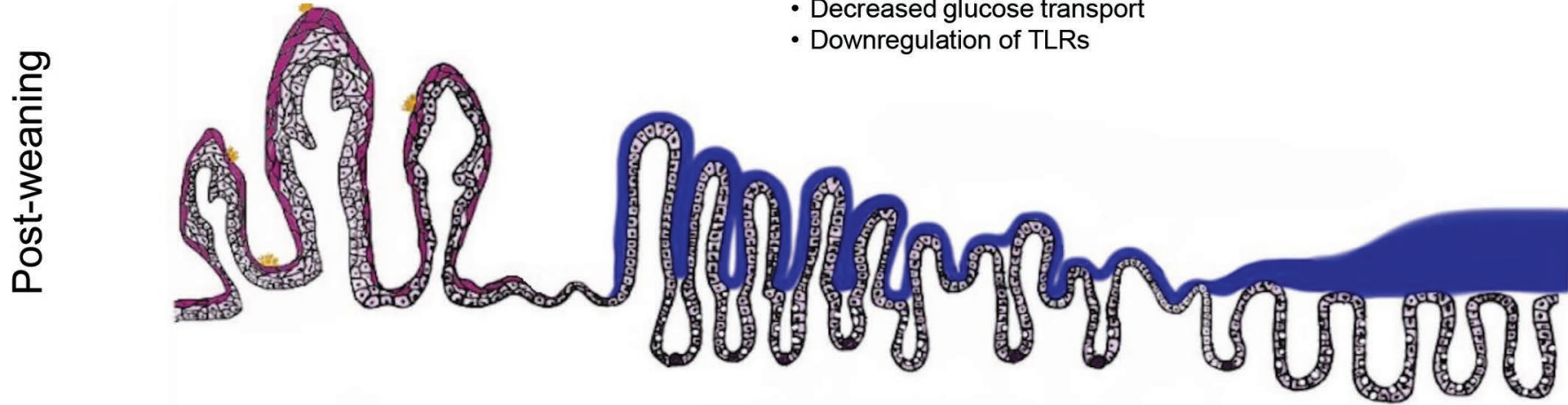

- Firmicutes: Bacteroidetes ratio increases

- Bacteroides decreases

- Bacteroides decreases and Prevotella increases

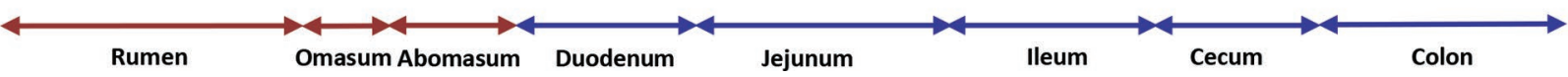

Figure 1. The diverse microbial and epithelial structure of the calf gastrointestinal tract pre- and postweaning. The majority of information available regarding the forestomach is limited to the rumen, and thus, unless otherwise stated in the figure, specific descriptions of gene function or microbial composition in the forestomach relate solely to the rumen. GLP-2 = glucagon-like peptide-2; TGF $\beta 1=$ transforming growth factor $\beta 1 ; \mathrm{TLR}=$ toll-like receptor. 
preweaning nutrition, but calves were not producing ketones at a rate similar to that of a mature ruminant until 60 d of age. Similarly, Bush (1988) found that calves were only at $40 \%$ the ketogenic rate of a mature ruminant at $30 \mathrm{~d}$ of age, despite being weaned at $28 \mathrm{~d}$ of age, underlining the limited capacity of the rumen to metabolize increases in VFAs resulting from ruminal fermentation at such a young age.

\section{MICROBIAL COLONIZATION OF THE FORESTOMACH}

Microbiological investigations of the calf's forestomach have focused primarily on the rumen, leaving a paucity of information regarding the omasum and abomasum. It has been considered that microbial inoculation of the newborn's rumen starts immediately after birth, through the vaginal canal, fecal material, colostrum, skin and saliva of the dam. However, members of typical functional ruminal populations, such as methanogens, fibrolytic bacteria, or Proteobacteria, have been detected in the rumen of calves less than 20 min after birth, suggesting that inoculation occurs even before birth (Guzman et al., 2015). Interestingly, these communities are already active as significant amounts of bacterial and archaeal RNA have been measured in the rumen of calves at birth (Malmuthuge et al., 2015). Rapid changes occur in the composition of the ruminal bacterial community during the first days of life (Jami et al., 2013) where the primo-colonizing bacteria appear to play an important role in shaping the biotope for strictly anaerobic microbial populations that colonize soon afterward (Fonty and Chaucheyras-Durand, 2007). Sequentially, establishment of aerobic or facultative anaerobic bacterial taxa occurs first in the rumen, where Proteobacteria- and Streptococcus-related sequences have been found in 1- to 3-d-old calves, but are rapidly supplanted by strictly anaerobic bacterial communities (Jami et al., 2013). Similarly, colonization of the rumen wall by epimural bacteria is also age related. Jiao et al. (2015) reported the phylum Proteobacteria was largely dominant on the rumen epithelium ( $>90 \%$ of sequences), with the majority belonging to the genus Escherichia in goat kids on the day of birth, which agrees with earlier studies using electron microscopy (Rieu et al., 1990). The quantitative importance of Proteobacteria within the epimural community in early life (Jiao et al., 2015; Wang et al., 2017), which has also been observed in adults (De Mulder et al., 2016), might have a particular role in scavenging oxygen diffusing from the capillary network (Cheng et al., 1979), thereby creating ecological conditions suitable for the establishment of anaerobic communities. The abundance of Proteobacteria associated with rumen epithelium decreases, and that of Firmicutes and Bacteroidetes increases with age (Jiao et al., 2015).

Cellulolytic bacteria and fungi are respectively detected after 1 and 2 wk of age (Fonty et al., 1987). Ciliate protozoa are detected from 2 to 3 wk of age, of which Entodinium sp. is the first to appear. Ciliates require the establishment of a complex microbiota beforehand (Fonty et al., 1988) and rearing conditions, such as separation from the mother at birth and artificial milk feeding, are detrimental to the optimal establishment of ruminal ciliates (Abecia et al., 2014; Yáñez-Ruiz et al., 2015). Studies with cultural methods have reported that the rumen microbial ecosystem is stabilized after 2 mo of life (Fonty et al., 1987). However, comparing rumen samples of calves of 6 mo and 2 years of age fed the same diet, Jami et al. (2013) observed that rumen microbiota was still significantly different, potentially indicating that at 6 mo of age the microbiota undergoes developmental changes independently of diet.

Although increasing animal age and the introduction of solid feed are expected to promote rumen bacterial diversity and species richness, with 45 to 47 bacterial genera belonging to 13 to 15 phyla being identified in 2- to 3-wk-old calves (Jami et al., 2013; Malmuthuge et al., 2014), recent characterization of the microbiome across weaning suggests otherwise, showing a reduction in microbial diversity in the weeks immediately following weaning (Meale et al., 2016). Sequencing of the 16S rRNA amplicon in ruminal microbiota of preweaned calves at $6 \mathrm{wk}$ of age and postweaned calves at $8 \mathrm{wk}$ of age, revealed a decline in operational taxonomic unitrichness and evenness (chao1 and Shannon indices, respectively) in postweaned calves (Meale et al., 2016). This could perhaps be due to extensive increases in fermentation of a single substrate and a low, fluctuating ruminal $\mathrm{pH}$ during the immediate postweaning period (Steele et al., 2015, 2016). It is possible that such a ruminal environment is less favorable for specific members of rumen microbiota, and thus, leads to reduced overall diversity, an effect that has also been observed following the addition of starch into the diet of Holstein cows (Zened et al., 2013). On the other hand, prolonged exposure to this environment could promote specific members of rumen microbiota that are capable of efficiently degrading a specific diet. As such, rumen microbial diversity may, in fact, increase over time when the microbiota becomes more mature and stable, similar to that described by Jami et al. (2013).

The age at which a calf is weaned can also dictate the nature of the microbial shift across weaning. Recently, we found (Meale et al., 2017) that calves weaned at a younger age (6 vs. 8 wk) showed a greater dissimilarity between pre- and postweaning than later weaned calves, who exhibited a gradual shift in between-group 
diversity as animals. This likely reflects the slower increase in starter consumption with age of later weaned calves, compared with the rapid compensatory increase in starter consumption in earlier weaned calves (Meale et al., 2017). Despite this, between-group diversity (determined using weighted UniFrac) was similar across all calves once they reached 9 wk of age, again resembling the convergence toward a mature state reported by Jami et al. (2013).

Recent studies (Li et al., 2012; Jami et al., 2013; Meale et al., 2016) suggest the developing gut of a preweaned calf contains the same dominant phyla, Bacteroidetes, Firmicutes, and Proteobacteria, as the more mature postweaned gut, although the relative abundances vary with age and stage of development. Typically, the relative abundance of Firmicutes increases postweaning (Jami et al., 2013; Meale et al., 2016, 2017). Conversely, the relative abundance of Bacteroidetes declines in calves at 5 versus 7 (Meale et al., 2017) wk of age and remains stable thereafter, indicating that weaning status is not the main driving factor of this shift and that perhaps this phylum is more responsive to the initiation of solid feed intake than the absence of milk in the diet. This is not the only ruminal taxon that displays a pattern of association with the level of starter consumption. For example, the relative abundance of Prevotella has been shown to reach a stable level once starter consumption rises above $100 \mathrm{~g}$ per $\mathrm{d}$, with the removal of milk from the diet having no effect on its abundance (Rey et al., 2014; Meale et al., 2016). Additionally, the relative abundance of Bacteroides showed a greater decline in earlier weaned calves consuming a higher ratio of MR to starter preweaning than calves weaned later and who were consuming a more moderate ratio of $\mathrm{MR}$ to starter preweaning (Meale et al., 2017). Collectively, these results, together with earlier studies (Hungate, 1966; Biesheuvel et al., 1991), indicate that the younger a calf begins to consume solid feed, the sooner a ruminal bacterial community more representative of a mature rumen develops. However, some taxa may not be as capable of handling a dramatic dietary change, such as when a calf is weaned before establishing a moderate intake of starter. As such, this represents a key area for future research, as we are largely unaware of the implications of preweaning GIT colonization on weaning stress and postweaning ruminal function.

\section{MOLECULAR ADAPTATIONS OF THE FORESTOMACH}

Although increasing information is available on the taxonomic composition of rumen microbiota, thanks to the development of amplicon sequencing techniques, data on the functional potential of microbiota in relation to animal age remain scarce. Rumen microbiota carries considerable functional diversity, as evidenced by the identification of more than 8,000 putative protein families from ruminal microbiota of preweaned calves ( $\mathrm{Li}$ et al., 2012). Microbial diversity within the rumen is associated with papillae length and rumen wall thickness, as well as the weight of reticulo-rumen, and finally, animal growth, as evidenced by animal models reared in sterile isolators and inoculated with more or less complex mixtures of rumen bacteria (Forano et al., 2010). Ecological conditions driving colonization of the rumen wall by epimural bacteria certainly play an important role in ruminal epithelial development and function (Malmuthuge et al., 2014). For instance, rumen epithelial expression of transforming growth factor $\beta 1$ (TGF $\beta 1$ ) and estrogen related receptor $\alpha(E S R R A)$, transcriptional regulators of rumen epithelial development and energy metabolism, are sensitive to butyrate (Connor et al., 2014), which might be provided by the genus Butyrivibrio, which was identified in more than $40 \%$ of bacterial sequences on rumen epithelium samples taken from 42-d-old goat kids (Jiao et al., 2015). Activation of such transcriptional regulators in the rumen may be dependent on diet composition, whereby activation of gene pathways participating in energy production, in which ESRRA may play a prominent role in FA absorption and metabolism, occurred in young calves fed MR supplemented with hay (Connor et al., 2014). Whereas supplementation of MR with a grain-based starter to preweaned calves upregulated expression of $T G F \beta 1$ mRNA and protein in rumen epithelium, compared with MR alone (Connor et al., 2014), and regardless of the inclusion of hay with calf starter in weaned calves, TGF 1 mRNA was the most highly upregulated gene in rumen epithelium of calves $3 \mathrm{wk}$ postweaning (11 wk of age; Kim et al., 2016). This, together with an additional activation of TGFBR 1 and downstream genes of the $T G F \beta 1$ intracellular pathway, suggests that cellular proliferation, recognition, differentiation, and apoptosis in the rumen are regulated by $T G F \beta 1$ during the weaning transition, and indicates a facilitatory role of $T G F \beta 1$ in rumen papillary differentiation when starter is consumed by the growing calf.

Chemical composition of the diet also seems to contribute to shifts in regulatory mechanisms in the rumen; for example, Naeem et al. (2012) observed a 7 -fold increase in expression of the nuclear receptor peroxisome proliferator-activated receptor delta $(P P A R D)$ in weaned calves fed elevated levels of CP preweaning, compared with a standard diet, indicating it has a key regulatory role in ruminal epithelial cell development when increased crude protein is supplied. As such, elucidating ligands of PPARD may provide the means for nutritional regulation of rumen development. These 
elevated dietary protein levels postweaning also increased expression of the growth-promoting gene IGF1, and increased reticulorumen mass, highlighting the importance of feeding high-quality starter as a means of providing metabolic fuel for postweaning ruminal development.

Furthermore, few studies have examined gene expression in the abomasum, where the expression of ghrelin, a regulator of growth hormone secretion, GIT development, and feed intake, increased in lambs from birth until reaching a plateau at $49 \mathrm{~d}$ of age (Wang et al., 2014), as would be expected to promote sufficient and rapid development of the abomasum with increasing age. However, a downregulation of abomasal ghrelin expression occurred at weaning indicating that perhaps the regulatory role of ghrelin is primarily relevant in the preweaning stage. Similarly, the provision of dietary starter may also affect the mechanistic regulation of feed intake in dairy calves as shown by the complete elimination of leptin mRNA expression, considered responsible for the long-term satiety effect, in both ruminal and abomasal tissue of 13-wk-old calves, an effect that was not observed in calves fed solely MR at 3 and 13 wk of age (Yonekura et al., 2002). The ability of starter to stimulate epithelial growth, combined with reductions in glucose oxidation, butyrate becoming the preferred oxidative substrate (Baldwin and Jesse, 1992), and increased production of ketone bodies, such as $\beta$-hydroxybutyrate, in rumen epithelial cells at weaning suggests that elimination of leptin expression in starterfed animals reflects a change in the satiety mechanism. Collectively, these studies suggest an overall shift in the molecular mechanisms regulating foregut development and functionality in calves, brought about by the functional and morphological changes experienced during the weaning transition.

\section{LOWER GUT ADAPTATIONS}

The lower gut is defined as the small intestine (duodenum, jejunum, and ileum), cecum, and colon. It harbors a diverse collection of cell types, such as absorptive epithelial cells, mucus-secreting cells (goblet cells), immune cells (Peyer's patches, Paneth cells, dendritic cells, and lymphocytes), and enteroendocrine cells that change in proportion and function within each region (Steel et al., 2015). Additionally, microbial diversity and richness increases from the proximal small intestine to the distal colon (Malmuthuge et al., 2015).

The first major adaption of the lower gut is to enable the transfer of Ig from colostrum to the calf, however, the mechanisms that regulate this transfer are rarely studied. Our current understanding is that after the first day of life, the gut undergoes a "closure," where large macromolecules, like Ig, are no longer able to be absorbed by the gut. When one considers the massive effect this gut closure has on calf immunity, it is surprising that we are not yet aware of the regulatory mechanisms orchestrating gut closure in calves. Studies conducted several decades ago in vitro and in small ruminant models (sheep and goats) have shown that Ig are not transported paracellularly (between cells) through the intestine, but are instead transported through enterocyte cells, in a process termed transcytosis (Jochims et al., 1994). The cellular process of transcytosis is thought to be initiated by a receptor-mediated pinocytosis (FcRn receptors; Hurley and Theil, 2011), which forms, inside a cell, an endosome full of large macromolecules, such as Ig. Once the endosome is within the enterocyte it has 3 possible pathways: (1) trafficking to basal members of the enterocyte, entering the lamina propria and eventually blood circulation (passive transfer), (2) recycled back to the lumen, or (3) metabolized by the epithelial cell (Hunziker and Kraehenbuhl, 1998). It is currently unknown if these pathways exist in the calf and the role nutrition and microbiology play in regulating Ig transfer through the GIT. It is also unknown how long passive transfer occurs, yet by d 5 to 7 of life, adult-type enterocytes contribute to the intestinal barrier, replacing the vacuolated fetal-type enterocytes (Guilloteau et al., 2009). It may be that the GIT is capable of Ig uptake for longer than $24 \mathrm{~h}$, which would promote the use of extended colostrum feeding schemes.

Although the passive transfer of maternal antibody is the main focus of colostrum management in neonatal calves, growth factors (insulin-like growth factor, $I G F-1$, and hormones) present in colostrum have also been shown to influence gut development (Baumrucker et al., 1994; Blättler et al., 2001). Feeding colostrum for the first $3 \mathrm{~d}$ of life increases small intestinal villus height and crypt depth of neonatal calves, compared with those fed MR. Correspondingly, the rate of epithelial cell proliferation increased with colostrum feeding compared with MR feeding (Blättler et al., 2001). It was noted that these effects were more prominent in the duodenum compared with that of the jejunum and ileum, which suggests that extending colostrum feeding may affect the proximal intestine more than the distal intestine.

During the first weeks of life, the GIT is continually sensing nutrient supply and communicating with other organs, such as the liver and pancreas, which contribute to digestion (i.e., hydrolysis and enzymatic breakdown). Pancreatic juice secretions increase until 4 wk of age (Guilloteau et al., 2009) and the $\mathrm{pH}$ at jejunal and ileal sites are rather constant between birth and 4 wk of age (Miyashige and Yahata, 1980; Guilloteau et al., 2009). 
Activity, site, and diversity of intestinal enzymes vary with age, feed, and feed intake (Guilloteau et al., 2009). Until weaning, hydrolysis of lactose and the subsequent absorption of glucose and galactose via the sodiumglucose cotransporter 1 are crucial for energy uptake. Accordingly, the abundance of this glucose transporter decreases with rumen development to a minimum in adult cows (Wood et al., 2000).

Although the effect of weaning on the rumen has received the bulk of attention in the literature, the lower gut also undergoes transformation during this time. It has recently been shown in dairy calves that weaning is associated with increased permeability of the GIT (Wood et al., 2015), suggesting integrity of the GIT may be compromised during this transition. In addition, it is possible that dairy calves suffer temporarily from hindgut acidosis stemming from incomplete digestion in the rumen, for they have shown elevated fecal starch levels during weaning (Eckert et al., 2015). This may lead to whole animal inflammation (Steele et al., 2016) and coincides with an increase in inflammatory markers in peripheral blood, such as the neutrophil to lymphocyte ratio, that has been reported during the early weaning of dairy calves at 6 wk of age (Kim et al., 2011), further emphasizing the importance of investigating the entire GIT, rather than the rumen in isolation. Additionally, it is important to note that the age of weaning may have influenced the inflammatory effect, whereas a later weaning may have lessened the inflammatory response; however, as this was not examined, no firm conclusions should be drawn. As such, the lower gut microbiological, structural, and functional changes require more attention and determining how these changes contribute to weaning stress is a logical next step in research.

\section{MICROBIAL ADAPTATIONS OF THE LOWER GUT}

As in the rumen, intestinal microbial colonization occurs from birth, and it is possible that inoculation comes from microbial bodies leaving the forestomach to reach the lower gut. However, the fact that several microbial representatives were found in the feces of calves less than 20 min after birth suggests that these organisms might start to colonize the GIT during delivery or even before birth (Guzman et al., 2015). Recently, a low-biomass microbiome has been characterized in the uterine cavity of nonpregnant women and was co-dominated by members of the phylum Bacteroidetes (Bacteroides xylanisolvens, Bacteroides fragilis, and Bacteroides thetaiotaomicron) and the genus Lactobacillus (Verstraelen et al., 2016). Moreover, bacteria from the maternal GIT have been detected and isolated in umbilical cord blood, amniotic fluid, meconium, and placental and fetal membranes without any clinical evidence of infection or inflammation in the mother-infant pair (Jimenez et al., 2005). The nature and abundance of microbial representatives in the lower gut are of primary importance as they represent a source of immunogenic molecules that will stimulate the development of animal defense mechanisms (YáñezRuiz et al., 2015). Studies on intestinal microbiota in newborn pre-ruminants have mostly focused on fecal microbiota as they are thought to be representative of the hindgut microbiota. In these studies, the main bacterial phyla were Bacteroidetes, Proteobacteria, and Firmicutes (Oikonomou et al., 2013; Meale et al., 2016), with the Bacteroides genus being strongly dominant, followed by Escherichia-Shigella and Faecalibacterium in Simmental calves (Klein-Jöbstl et al., 2014). In 1-wkold Holstein calves, active members of the BacteroidesPrevotella group, Faecalibacterium, Clostridium coccoides-Eubacterium rectale group, and Atopobium cluster were detected at high levels in the feces (Uyeno et al., 2010). Throughout a 12-wk period, rRNA of Bacteroides-Prevotella and $\mathrm{Cl}$. coccoides-Eu. rectale groups constituted the major fraction of microbiota (50 to $70 \%$ ). Malmuthuge et al. (2014) monitored composition of the bacteria along the GIT (rumen, jejunum, ileum, cecum, and colon) in 3-wk-old preweaned bull calves. They observed clear regional variations in bacterial composition, with high prevalence of Bacteroidetes in the rumen contents and in the large intestine, and high prevalence of Firmicutes in the small intestine. Notably, a high abundance in the genus Sharpea was measured in the jejuno-ileal section, which may be linked to its capacity to uptake and ferment milk sugars. In the colon of 2- or 4-wk-old preweaned Holstein calves, Castro et al. (2016) found Firmicutes and Actinobacteria as the most abundant phyla, with high dominance of Lactobacillus genus, and an almost unique bacterial assemblage (Lactobacillus-Bifidobacterium-Streptococcus) feature of the colon. Uyeno et al. (2010) studied the evolution of fecal bacteria from birth to $12 \mathrm{wk}$ of life and reported that fecal active fiber degraders such as Ruminococcus and Fibrobacter only emerged at detectable levels ( 1 to $2 \%$ ) postweaning. However, it is important to note that these calves were fed MR containing antibiotics, colistin and bacitracin, which target both gram-positive and negative bacteria, and thus, may have prevented the earlier appearance of these fiber degraders.

Microbial diversity of the lower gut increases throughout the preweaning period with noted association between increased weight gain and higher diversity after the second week of life. However, incidence of disease and antibiotic treatment can decrease diversity having negative effects on calf weight. For example, the Chao1 index was shown to gradually increase from wk 1 to 7 
of life in Holstein dairy calves (Oikonomou et al., 2013). Yet, a decrease was noted during the third, fourth, and fifth week of life in calves that suffered from pneumonia and were treated with antibiotics. Diarrhea incidence during the first 4 wk of the calves' life was also associated with a reduction of microbial diversity during the third week of life, despite no antibiotic treatment, suggesting that the disease state itself can also reduce microbial diversity. In comparisons of pre- and postweaned calves, fecal microbiota showed greater richness and evenness postweaning (Meale et al., 2016), possibly due to a higher intake of solid feed postweaning, which would increase the amount of substrates that pass to the hindgut, where environmental conditions are more stable. However, despite this, 2 studies that have examined the shift in intestinal taxa over the weaning transition in dairy calves did not observe changes in the relative abundance of the 3 most prevalent intestinal phyla: Firmicutes, Bacteroidetes, and Proteobacteria (Meale et al., 2016, 2017). As is the case in most commercial settings, the calves in these studies had access to starter from the first weeks of life, meaning that at the time of first sampling starter consumption was in excess of $50 \mathrm{~g}$ per $\mathrm{d}$, making it reasonable to conclude that a shift in Bacteroidetes may have already occurred. The dietary balance between carbohydrates and protein or animal fat is thought to be the primary factor shifting fecal microbiota, where greater Bacteroides abundance is associated with high protein and animal fat diets, compared with Prevotella, which predominates with greater carbohydrate intake (Wu et al., 2012; Thompson et al., 2015), especially those rich in starch and plant polysaccharides. Thus, a shift in the source of dietary nutrients away from MR and toward that of a high fiber diet, as experienced during weaning, expectedly reduced the relative abundance of fecal Bacteroides (Meale et al., 2016) and increased that of Prevotella (Meale et al., 2016, 2017).

\section{MOLECULAR ADAPTATIONS OF THE LOWER GUT}

The underpinning molecular mechanisms regulating epithelial cell proliferation, differentiation, and the subsequent changes in the lower gut brought about by the weaning transition, are not well established in the calf. Studies have attempted to identify specific mechanisms of regulation involved in epithelial growth, with several focusing on the stimulatory role of the short-chain fatty acid butyrate or its sodium salt. Supplementation of protected butyrate with MR in calves increased plasma glucagon-like peptide-2 (GLP-2) and IGF-1, and was associated with increased levels of proliferation and growth of the intestine and also of the rumen (Górka et al., 2009, 2011). The degree to which GLP-2 plays a regulatory role in GIT development varies according to location, but is considered to play only a minor role in forestomach development, due to its negligible expression in the rumen (Taylor-Edwards et al., 2010). Conversely, it is considered an important stimulator of intestinal development, where calves fed calf starter supplemented with $0.6 \%$ sodium butyrate encapsulated within a triglyceride matrix had heavier small intestines. Subsequent links between small intestine weight and reticulorumen weight have been reported in newborn calves (Górka et al., 2011), suggesting that protected butyrate supplementation could increase small intestinal growth, with an additional indirect stimulation of rumen development, via the regulatory effects of plasma GLP-2. Moreover, a shift at weaning was observed in duodenal leptin mRNA expression in calves (Yonekura et al., 2002). In contrast to the rumen, expression was observed in preweaned calves aged $3 \mathrm{wk}$ and weaned calves at $13 \mathrm{wk}$, even in the presence of starter and VFA supplementation of MR diets suggesting that similar to gastric leptin in nonruminant animals, duodenal leptin may have a role in short-term regulation of food intake (Cinti et al., 2000).

Collectively, these studies suggest an overall shift in the regulatory mechanisms governing GIT development and functionality, coinciding with associated shifts in microbiota that occur during the weaning transition. Thus, it is essential for future studies to closely examine the intimate cross-talk between gut microorganisms, diet, and the host. One such study by Liang et al. (2014) found region-specific miRNA (small molecules of RNA involved in regulation processes of GIT development) in different sections of the calf intestinal epithelium, the expression patterns of which changed throughout the first 6 wk of life, with the most notable changes being observed during the first week. Many of these miRNA targeted genes are involved in the immune response, suggesting that miRNA could regulate immune function development during early life. Relationships between the expression of miRNA and the abundance of beneficial bacteria (Lactobacillus, Bifidobacterium) were observed, highlighting the potential role of miRNA in cross-talk between host and gut microbiota.

Similarly, the specific interaction between the intestinal microbiome and immune response of intestinal epithelium was examined by Malmuthuge et al. (2012) in pre- (3 wk of age) and postweaned ( 6 wk of age) Holstein bull calves. The authors quantified the expression of toll-like receptors (TLR), peptidoglycan recognition proteins (PGLYRP1), and antimicrobial peptides ( $\beta$-defensin) using quantitative PCR. A comparison of TLR (TLR1-TLR10) expression in the rumen, jejunum, ileum, cecum, and colon revealed that most TLR were significantly downregulated after weaning, 
coinciding with a drastic change in the microbiome, as well as the morphological and functional changes in the GIT described above. Therefore, the authors suggest that the downregulation of TLR in weaned calves may reflect a developmental shift toward that of a mature ruminant to prevent unnecessary inflammatory responses to a shifting intestinal microbiome. Similarly, Malmuthuge et al. (2013) focused on the start of the weaning transition (i.e., once calves consume over 680 $\mathrm{g}$ starter per d for 3 consecutive days) compared with a milk only diet on intestinal expression of TLR6 and TLR2, the latter of which is typically upregulated in humans with inflammatory bowel syndrome. Calves consuming starter showed an upregulation of these genes, indicating starter feeding may have an influence on gut permeability by allowing more bacterial products to breach the mucosal barrier and stimulate the expression of host pattern recognition receptors.

In the absence of further transcriptomic studies exploring pre- and postweaning differences in dairy calves we employed the use of a bioinformatics software (PICRUSt; Langille et al., 2013) to predict metagenome functional content from a marker gene, in our case 16S rRNA across weaning (Meale et al., 2017). We noted a significant reduction in genes associated with AA, energy, and carbohydrate metabolism in the fecal microbiome of weaned calves, which has been considered to be representative of the intestinal microbiome. More specifically, within carbohydrate metabolism, the number of inferred genes assigned to the fructose and mannose metabolism pathways, as well as the starch and sucrose metabolism pathways decreased postweaning, reflecting the shift in nutrient metabolism away from the lower GIT to ruminal fermentation. Although informative, these data are only predictive, and thus, it is clear that further studies need to focus on the shifting transcriptome of calves throughout the weaning transition, and not just its initiation, to determine how the calf responds to such a large change in digestive and absorptive functions.

\section{FUTURE DIRECTIONS}

As described above, many factors can affect the transition experienced by the GIT up to and throughout the weaning process. Although molecular level changes have been detected together with gut physiological and environmental change, the regulatory mechanisms are still largely unknown, especially in regard to the factors controlling host-microbial interactions throughout the GIT. The effect of host genetics on the establishment of individualized gut microbiota in mammals has been recently reported (Benson et al., 2010; BuhnikRosenblau et al., 2011; Lim et al., 2016), suggesting that the host plays an important role in selecting the residential microbiota to suit its physiological and functional purposes. Although the effect of the hosts on gut microbiota has attracted attention, studies have mostly been conducted in humans or using murine models. The recent efforts to link the cattle sire breed (Hernandez-Sanabria et al., 2013) and host gene SNP (Roehe et al., 2016) with rumen microbiota indicate the importance of understanding host genetics in regulating microbial colonization, as well as gene expression. In addition, individual variation in the calf's ability to adapt preweaning and during the weaning process has not been studied. Weaning stress could be a phenotypic trait that could be partially attributed to genetic variation, in addition to management and nutritional strategies. Future research on calf genotyping with varied performances in response to weaning stress may lead to selection and breeding tools for animals with a greater adaptability. In addition, heritability of microbiota associated with adaptation to weaning should be taken into account, as low heritability microbiota may not be good markers for selection. Moreover, it is commonly accepted that the gut microbiota can be affected by host, environment (diet), and microbe-microbe interactions. How the microbial interactions affect microbial colonization and the subsequent effects on host function should be an emerging research area. Regardless, comprehensive data collection on animal performance, genetics, gene expression, and microbial composition and function from large cohorts of animals is needed to dissect the mechanisms involved, which form the basis of knowledge for future manipulations and enhancement of animal performance during the weaning process.

\section{CONCLUSIONS}

It has become clear over the last decade that the nutrition and health of calves in early life can have longstanding effects on their growth, development, and productivity. Gut development, especially gut closure and weaning, is one of the most dramatic transformations in nature. Yet, an understanding of how commonly practiced nutritional protocols can affect gut development has not been achieved. In particular, few studies have directly investigated the influence of prenatal nutrition, colostrum feeding frequency and duration, preweaning level of nutrition, and weaning duration and age on gut health and development. Although it is necessary from a production standpoint to measure growth during the evaluation of early-life nutrition programs, there is also great value in determining the dietary effect on gut development to advance our understanding in this field. Expanding our knowledge using high-throughput sequencing technologies has been useful in this regard, 
however, we cannot lose sight of nutrition fundamentals and the effect they have on digestion, physiology, and ultimately application in our industry.

\section{ACKNOWLEDGMENTS}

The authors thank the support of the Natural Science and Engineering Research Council (Ottawa, ON, Canada) and Alberta Milk (Edmonton, AB, Canada). We also appreciate the dedication of Jade Pyo (University of Alberta, Canada) for her assistance with the illustration in this review.

\section{REFERENCES}

Abecia, L., E. Ramos-Morales, G. Martinez-Fernandez, A. Arco, A. I. Martin-Garcia, C. J. Newbold, and D. Yáñez-Ruiz. 2014. Feeding management in early life influences microbial colonization and fermentation in the rumen of newborn goat kids. Anim. Prod. Sci. 54:1449-1454

Ahmed, A. F., P. D. Constable, and N. A. Misk. 2002. Effect of feeding frequency and route of administration on abomasal luminal $\mathrm{pH}$ in dairy calves fed milk replacer. J. Dairy Sci. 85:1502-1508.

Allen, M. S. 1997. Relationship between fermentation acid production in the rumen and the requirement for physically effective fiber. J. Dairy Sci. 80:1447-1462.

Anderson, K. L., T. G. Nagaraja, J. L. Morrill, T. B. Avery, S. J. Galitzer, and J. E. Boyer. 1987. Ruminal microbial development in conventionally or early-weaned calves. J. Anim. Sci. 64:1215-1226.

Bach, A., L. Domingo, C. Montoro, and M. Terre. 2013. Short communication: Insulin responsiveness is affected by the level of milk replacer offered to young calves. J. Dairy Sci. 96:4634-4637.

Baldwin, R. L., VI, and B. W. Jesse. 1992. Developmental changes in glucose and butyrate metabolism by isolated sheep rumen epithelial cells. J. Nutr. 122:1149-1153.

Baldwin, R. L., VI, K. R. McLeod, J. L. Klotz, and R. N. Heitmann. 2004. Rumen development, intestinal growth and hepatic metabolism metabolism in the pre- and postweaning ruminant. J. Dairy Sci. 87(E. Suppl.):E55-E65.

Baumrucker, C. R., D. L. Hadsell, and J. W. Blum. 1994. Effects of dietary insulin-like growth factor 1 on growth and insulin-like growth factor receptors in neonatal calf intestine. J. Anim. Sci. $72: 428-433$.

Beharka, A. A., T. G. Nagaraja, and J. L. Morrill. 1991. Performance and ruminal function development of young calves fed diets with Aspergillus oryzae fermentation extract. J. Dairy Sci. 74:43264336.

Benson, A. K., S. A. Kelly, R. Legge, F. Ma, S. J. Low, J. Kim, M. Zhang, P. L. Oh, D. Nehrenberg, K. Hua, S. D. Kachman, E. N. Moriyama, J. Walter, D. A. Peterson, and D. Pomp. 2010. Individuality in gut microbiota composition is a complex polygenic trait shaped by multiple environmental and host genetic factors. Proc. Natl. Acad. Sci. USA 107:18933-18938.

Berends, H. 2014. Nutrient utilization, dietary preferences, and gastrointestinal development in veal calves: Interactions between solid feed and milk replacer. PhD thesis. Wageningen University, Wageningen, the Netherlands.

Biesheuvel, M. H., P. G. H. Bijker, and H. A. P. Urlings. 1991. Some aspects of the gastrointestinal microflora of veal calves fed different rations-A pilot-study. Vet. Q. 13:97-104.

Blättler, U., H. M. Hammon, C. Morel, C. Philipona, A. Rauprich, V. Romé, I. Le Huërou-Luron, P. Guilloteau, and J. W. Blum. 2001. Feeding colostrum, its composition and feeding duration variably modify proliferation and morphology of the intestine and digestive enzyme activities of neonatal calves. J. Nutr. 131:1256-1263.
Buhnik-Rosenblau, K., Y. Danin-Poleg, and Y. Kashi. 2011. Predominant effect of host genetics on levels of Lactobacillus johnsonii bacteria in the mouse gut. Appl. Environ. Microbiol. 77:6531-6538.

Bush, R. S. 1988. Effect of age and diet on in vitro metabolism in rumen epithelium from Holstein calves. Can. J. Anim. Sci. 68:12451251.

Cant, J. P., B. W. McBride, and W. J. Croom Jr.. 1996. The regulation of intestinal metabolism and its impact on whole animal energetics. J. Anim. Sci. 74:2541-2553.

Castro, J. J., A. Gomez, B. A. White, J. R. Loften, and J. K. Drackley. 2016. Changes in the intestinal bacterial community, short-chain fatty acid profile, and intestinal development of preweaned Holstein calves. 2. Effects of gastrointestinal site and age. J. Dairy Sci. 99:9703-9715.

Cheng, K. J., R. P. McCowan, and J. W. Costerton. 1979. Adherent epithelial bacteria in ruminants and their roles in digestive tract function. Am. J. Clin. Nutr. 32:139-148.

Cinti, S., R. D. Matteis, C. Pico, E. Ceresi, A. Obrador, C. Maffeis, J. Oliver, and A. Palou. 2000. Secretory granules of endocrine and chief cells of human stomach mucosa contain leptin. Int. J. Obes. Relat. Metab. Disord. 24:789-793.

Connor, E. E., R. L. Baldwin VI, M. P. Walker, S. E. Ellis, C. Li, S. Kahl, H. Chung, and R. W. Li. 2014. Transcriptional regulators transforming growth factor- $\beta 1$ and estrogen-related receptor- $\alpha$ identified as putative mediators of calf rumen epithelial tissue development and function during weaning. J. Dairy Sci. 97:41934207.

De Mulder, T., K. Goossens, N. Peiren, L. Vandaele, A. Haegeman, C. De Tender, T. Ruttink, T. V. de Wiele, and S. De Campeneere. 2016. Exploring the methanogen and bacterial communities of rumen environments: Solid adherent, fluid and epimural. FEMS Microbiol. Ecol. 93:fiw215. https://doi.org/10.1093/femsec/fiw251.

Eckert, E., H. E. Brown, K. E. Leslie, T. J. DeVries, and M. A. Steele. 2015. Weaning age affects growth, feed intake, gastrointestinal development, and behavior in Holstein calves fed an elevated plane of nutrition during the preweaning stage. J. Dairy Sci. 98:6315-6326.

Ellingsen, K., C. M. Mejdell, N. Ottesen, S. Larsen, and A. M. Grondahl. 2016. The effect of large milk meals on digestive physiology and behavior in dairy calves. Physiol. Behav. 154:169-174.

Fonty, G., and F. Chaucheyras-Durand. 2007. Les écosystèmes digestifs. Ed Tec and Doc Lavoisier, Paris, France.

Fonty, G., P. Gouet, J. P. Jouany, and J. Senaud. 1987. Establishment of the microflora and anaerobic fungi in the rumen of lambs. J. Gen. Microbiol. 133:1835-1843.

Fonty, G., J. Senaud, J. P. Jouany, and P. Gouet. 1988. Establishment of ciliate protozoa in the rumen of conventional and conventionalized lambs: Influence of diet and management conditions. Can. J. Microbiol. 34:235-241.

Forano, E., F. Chaucheyras-Durand, and G. Fonty. 2010. Structuration de l'écosystème ruminal chez le nouveau-né: Influence de facteurs écologiques. Bull. GTV 52:13-19.

Furness, J. B., L. R. Rivera, H. J. Cho, D. M. Bravo, and B. Callaghan. 2013. The gut as a sensory organ. Nat. Rev. Gastroenterol. Hepatol. 10:729-740. https://doi.org/10.1038/nrgastro.2013.180.

Gäbel, G., J. R. Aschenbach, and F. Müller. 2002. Transfer of energy substrates across the ruminal epithelium: Implications and limitations. Anim. Health Res. Rev. 3:15-30.

Górka, P., Z. M. Kowalski, P. Pietrzak, A. Kotunia, W. Jagusiak, J. J. Holst, P. Guilloteau, and R. Zabielski. 2011. Effect of method of delivery of sodium butyrate on rumen development in newborn calves. J. Dairy Sci. 94:5578-5588.

Górka, P., Z. M. Kowalski, P. Pietrzak, A. Kotunia, R. Kiljanczyk, J. Flaga, J. J. Holst, P. Guilloteau, and R. Zabielski. 2009. Effect of sodium butyrate supplementation in milk replacer and started diet on rumen development in calves. J. Physiol. Pharmacol. 60:47-53.

Guilloteau, P., O. Zabielski, and J. W. Blum. 2009. Gastrointestinal tract and digestion in the young ruminant: Ontogenesis, adaptions, consequences and manipulations. J. Physiol. Pharmacol. 60:37-46.

Guzman, C. E., L. T. Bereza-Malcolm, B. De Groef, and A. E. Franks. 2015. Presence of selected methanogens, fibrolytic bacteria, and 
Proteobacteria in the gastrointestinal tract of neonatal dairy calves from birth to 72 hours. PLoS One 10:e0133048.

Hernandez-Sanabria, E., L. A. Goonewardene, Z. Wang, M. Zhou, S. S. Moore, and L. L. Guan. 2013. Influence of sire breed on the interplay among rumen microbial populations inhabiting the rumen liquid of the progeny in beef cattle. PLoS One 8:e58461.

Hodgson, J. 1971. The development of solid food intake in calves. 4. The effect of the addition of material to the rumen, or its removal from the rumen, on voluntary food intake. Anim. Prod. 13:581-592.

Hungate, R. E. 1966. The Rumen and Its Microbes. 3rd ed. Academic Press, New York, NY.

Hunziker, W., and J. P. Kraehenbuhl. 1998. Epithelial transcytosis of immunoglobulins. J. Mammary Gland Biol. Neoplasia 3:287-302.

Hurley, W. L., and P. K. Theil. 2011. Perspective on immunoglobulins in colostrum and milk. Nutrients 3:442-474.

Jami, E., A. Israel, A. Kotser, and I. Mizrahi. 2013. Exploring the bovine rumen bacterial community from birth to adulthood. ISME J. 7:1069-1079.

Jiao, J., J. Huang, C. Zhou, and Z. Tan. 2015. Taxonomic identification of ruminal epithelial bacterial diversity during rumen development in goats. Appl. Environ. Microbiol. 81:3502-3509.

Jimenez, E., L. Fernandez, M. L. Marin, R. Martin, J. M. Odriozola, C. Nueno-Palop, A. Narbad, M. Olivares, J. Xaus, and J. M. Rodriguez. 2005. Insolation of commensal bacteria from umbilical cord blood of health neonates by caesarean section. Curr. Microbiol. 51:270-274.

Jochims, K., F. J. Kaup, and D. Drommer. 1994. An immunoelectron microscopic investigation of colostral IgG absorption across the intestine of newborn calves. Res. Vet. Sci. 57:75-80.

Khan, M. A., A. Bach, D. M. Weary, and M. A. G. von Keyserlingk. 2016. Invited review: Transitioning from milk to solid feed in dairy heifers. J. Dairy Sci. 99:885-902.

Khan, M. A., D. M. Weary, and M. A. G. von Keyserlingk. 2011. Invited review: Effects of milk ration on solid feed intake, weaning, and performance in dairy heifers. J. Dairy Sci. 94:1071-1081.

Kim, M. H., J. Yang, S. D. Upadhaya, H. Lee, C. Yun, and J. K. Ha. 2011. The stress of weaning influences serum levels of acute-phase proteins, iron-binding proteins, inflammatory cytokines, cortisol, and leukocyte subsets in Holstein calves. J. Vet. Sci. 12:151-157.

Kim, Y.-H., N. Touji, K. Kizaki, S. Kushibiki, T. Ichijo, and S. Sato. 2016. Effects of dietary forage and calf starter on ruminal $\mathrm{pH}$ and transcriptomic adaptation of the rumen epithelium in Holstein calves during the weaning transition. Physiol. Genomics 48:803809. https://doi.org/10.1152/physiolgenomics.00086.2016.

Klein-Jöbstl, D., E. Schornsteiner, E. Mann, M. Wagner, M. Drillich and S. Schmitz-Esser. 2014. Pyrosequencing reveals diverse fecal microbiota in Simmental calves during early development. Front. Microbiol. 5:622. https://doi.org/10.3389/fmicb.2014.00622.

Kmet, V., K. Boda, P. Javorský, and R. Nemcová. 1986. The enzymatic activity of rumen microflora in calves. J. Anim. Physiol. Anim. Nutr. (Berl.) 56:73-77.

Langille, M. G. I., J. Zaneveld, J. G. Caporaso, D. McDonald, D. Knights, J. A. Reyes, J. C. Clemente, D. E. Burkepile, R. L. Vega Thurber, R. Knight, R. G. Beiko, and C. Huttenhower. 2013. Predictive functional profiling of microbial communities using $16 \mathrm{~S}$ rRNA marker gene sequences. Nat. Biotechnol. 31:814-821.

Li, R. W., E. E. Connor, C. Li, R. L. V. I. Baldwin, and M. E. Sparks. 2012. Characterization of the rumen microbiota of pre-ruminant calves using metagenomic tools. Environ. Microbiol. 14:129-139.

Liang, G., N. Malmuthuge, T. B. McFadden, H. Bao, P. J. Griebel, P. Stothard, and L. L. Guan. 2014. Potential regulatory role of microRNAs in the development of bovine gastrointestinal tract during early life. PLoS One 9:e92592.

Lim, M. Y., H. J. You, H. S. Yoon, B. Kwon, J. Y. Lee, S. Lee, Y.-M Song, K. Lee, J. Sung, and G. Ko. 2016. The effect of heritability and host genetics on the gut microbiota and metabolic syndrome. Gut https://doi.org/10.1136/gutjnl-2015-311326. In press.

MacPherson, J. A. R., H. Berends, L. N. Leal, J. P. Cant, J. MartinTereso, and M. A. Steele. 2016. Effect of plane of nutrition and age on glucose and insulin kinetics and abomasal emptying in female Holstein dairy calves fed twice daily. J. Dairy Sci. 99:8007-8017.

Malmuthuge, N., P. J. Griebel, and L. L. Guan. 2014. Taxonomic identification of commensal bacteria associated with the mucosa and digesta throughout the gastrointestinal tracts of preweaned calves. Appl. Environ. Microbiol. 80:2021-2028.

Malmuthuge, N., P. J. Griebel, and L. L. Guan. 2015. The gut microbiome and its potential role in the development and function of newborn calf gastrointestinal tract. Front. Vet. Sci. 2:36.

Malmuthuge, N., M. Li, P. Fries, P. J. Griebel, and L. L. Guan. 2012. Regional and age dependent changes in gene expression of Tolllike receptors and key antimicrobial defence molecules throughout the gastrointestinal tract of dairy calves. Vet. Immunol. Immunopathol. 146:18-26.

Malmuthuge, N., M. Li, L. A. Goonewardene, M. Oba, and L. L. Guan. 2013. Effect of calf starter feeding on gut microbial diversity and expression of genes involved in host immune responses and tight junctions in dairy calves during weaning transition. J. Dairy Sci. 96:3189-3200.

Marshall, T. S. 2009. Abomasal ulceration and tympany of calves. Vet. Clin. Food. Anim. 25:209-220.

Meale, S. J., S. Li, P. Azevedo, H. Derakhshani, J. C. Plaizier, E. Khafipour, and M. A. Steele. 2016. Development of ruminal and fecal microbiomes are affected by weaning but not weaning strategy in dairy calves. Front. Microbiol. 7:582.

Meale, S. J., S. C. Li, P. Azevedo, H. Derakhshani, T. J. De Vries, J. C. Plaizier, M. A. Steele, and E. Khafipour. 2017. Weaning age influences the severity of gastrointestinal microbiome shifts in dairy calves. Sci. Rep. In press.

Miyashige, T., and S. Yahata. 1980. Development of intestinal disaccharase activities in nursing calves. Jpn. J. Zootech. Sci. 51:58-68.

Naeem, A., J. K. Drackley, J. Stamey, and J. J. Loor. 2012. Role of metabolic and cellular proliferation genes in ruminal development in response to enhanced plane of nutrition in neonatal Holstein calves. J. Dairy Sci. 95:1807-1820.

Oikonomou, G., A. G. V. Teixeira, C. Foditsch, M. L. Bicalho, V. S. Machado, and R. C. Bicalho. 2013. Fecal microbial diversity in pre-weaned dairy calves as described by pyrosequencing of metagenomic 16S rDNA. Associations of Faecalibacterium species with health and growth. PLoS One 8:e63157.

Rey, M., F. Enjalbert, S. Combes, L. Cauquil, O. Bouchez, and V. Monteils. 2014. Establishment of ruminal bacterial community in dairy calves from birth to weaning is sequential. J. Appl. Microbiol. 116:245-257.

Rey, M., F. Enjalbert, and V. Monteils. 2012. Establishment of ruminal enzyme activities and fermentation capacity in dairy calves from birth through weaning. J. Dairy Sci. 95:1500-1512.

Rieu, F., G. Fonty, B. Gaillard, and P. Gouet. 1990. Electron microscopy study of the bacteria adherent to the rumen wall in young conventional lambs. Can. J. Microbiol. 36:140-144.

Roehe, R., R. J. Dewhurst, C.-A. Duthie, J. A. Rooke, N. McKain, D. W. Ross, J. J. Hyslop, A. Waterhouse, T. C. Freeman, M. Watson, and R. J. Wallace. 2016. Bovine host genetic variation influences rumen microbial methane production with best selection criterion for low methane emitting and efficiently feed converting hosts based on metagenomic gene abundance. PLoS Genet. 12:e1005846.

Sahoo, A., D. N. Kamra, and N. N. Pathak. 2005. Pre- and postweaning attributes in faunated and ciliate-free calves fed calf starter with or without fish meal. J. Dairy Sci. 88:2027-2036.

Sander, E. G., R. G. Warner, H. N. Harrison, and J. K. Loosli. 1959. The stimulatory effect of sodium butyrate and sodium propionate on the development of rumen mucosa in the young calf. J. Dairy Sci. 42:1600-1605.

Stahel, P., J. P. Cant, J. A. R. MacPherson, and M. A. Steele. 2016. A mechanistic model of intermittent gastric emptying and glucoseinsulin dynamics following a meal containing milk components. PLoS One 11:e0156443.

Steele, M. A., L. E. Leal, F. Soberon, J. H. Doelman, M. Carson, and J. A. Metcalf. 2015. Gradual weaning affects pre and post-weaning feed intake, growth and gastrointestinal development in Holstein 
calves fed an elevated plane of nutrition during the pre-weaning period. J. Anim. Sci. 98:242. (Abstr.)

Steele, M. A., G. B. Penner, F. Chaucheyras-Durand, and L. L. Guan. 2016. Development and physiology of the rumen and the lower gut: Targets for improving gut health. J. Dairy Sci. 99:4955-4966.

Stobo, I. J., J. H. Roy, and H. J. Gaston. 1966. Rumen development in the calf. 1 . The effect of diets containing different proportions of concentrates to hay on rumen development. Br. J. Nutr. 20:171188.

Suárez, B., C. Van Reenen, W. Gerrits, N. Stockhofe-Zurwieden, A. Van Vuuren, and J. Dijkstra. 2006. Effects of supplementing concentrates differing in carbohydrate composition in veal calf diets: II. Rumen development. J. Dairy Sci. 89:4376-4386.

Tamate, H., A. D. McGilliard, N. L. Jacobson, and R. Getty. 1962. Effect of various dietaries on the anatomical development of the stomach in the calf. J. Dairy Sci. 45:408-420.

Taylor-Edwards, C. C., D. G. Burrin, J. C. Matthews, K. R. McLeod, J. J. Holst, and D. L. Harmon. 2010. Expression of mRNA for proglucagon and glucagon-like peptide-2 (GLP-2) receptor in the ruminant gastrointestinal tract and the influence on energy intake. Domest. Anim. Endocrinol. 39:181-193.

Thompson, A. L., A. Monteagudo-Mera, M. B. Cadenas, M. L. Lampl, and M. A. Azcarate-Peril. 2015. Milk- and solid-feeding practices and daycare attendance are associated with differences in bacterial diversity, predominant communities, and metabolic and immune function of the infant gut microbiome. Front. Cell. Infect. Microbiol. 5:3 https://doi.org/10.3389/fcimb.2015.00003.

USDA. 2007. NAHMS Dairy Part 1: Reference of dairy cattle health and management practices in the United States, 2007. USDA, Washington, DC.

Uyeno, Y., Y. Sekiguchi, and Y. Kamagata. 2010. rRNA-based analysis to monitor succession of faecal bacterial communities in Holstein calves. Lett. Appl. Microbiol. 51:570-577.

Verstraelen, H., R. Vilchez-Vargas, F. Desimpel, R. Jauregui, N. Vankeirsbilck, S. Weyers, R. Verhelst, P. De Sutter, D. H. Pieper, and T. Van De Wiele. 2016. Characterization of the human uterine microbiome in non-pregnant women through deep sequencing of the V1-2 region of the 16S rRNA gene. PeerJ 4:e1602. https://doi. org/10.7717/peerj.1602.
Wang, W., L. Cheng, J. Guo, Y. Ma, and F. Li. 2014. Expression of Ghrelin in gastrointestinal tract and the effect of early weaning on Ghrelin expression in lambs. Mol. Biol. Rep. 41:909-914.

Wang, Z., C. Elekwachi, J. Jiao, M. Wang, S. Tang, C. Zhou, Z. Tan, and R. J. Forster. 2017. Changes in metabolically active bacterial community during rumen development, and their alteration by rhubarb root powder revealed by $16 \mathrm{~S}$ rRNA amplicon sequencing. Front. Microbiol. 8:159.

Warner, R. G., W. P. Flatt, and J. K. Loosli. 1965. Dietary factors influencing the development of the ruminant stomach. Agric. Food Chem. 4:788-792.

Windeyer, M. C., K. E. Lesliea, S. M. Godden, D. C. Hodgins, K. D. Lissemore, and S. J. LeBlanc. 2014. Factors associated with morbidity, mortality, and growth of dairy heifer calves up to 3 months of age. Prev. Vet. Med. 113:231-240.

Wood, I. S., J. Dyer, R. R. Hofmann, and S. P. Shirazi-Beechey. 2000. Expression of the Na+/glucose co- transporter (SGLT1) in the intestine of domestic and wild ruminants. Eur. J. Phys. 441:155-162.

Wood, K. M., S. I. Palmer, M. A. Steele, J. A. Metcalf, and G. B. Penner. 2015. The influence of age and weaning on permeability of the gastrointestinal tract in Holstein bull calves. J. Dairy Sci. 98:7226-7237.

Wu, S., R. L. V. I. Baldwin, W. Li, C. Li, E. E. Connor, and R. W. Li. 2012. The bacterial community composition of the bovine rumen detected using pyrosequencing of $16 \mathrm{~S}$ rRNA genes. Metagenomics (Cairo) 1:1-11.

Yáñez-Ruiz, D. R., L. Abecia, and C. J. Newbold. 2015. Manipulating rumen microbiome and fermentation through interventions during early life: A review. Front. Microbiol. 6:1133. https://doi. org/10.3389/fmicb.2015.01133.

Yonekura, S., K. Kitade, G. Furukawa, K. Takahashi, N. Katsumata, K. Katoh, and Y. Obara. 2002. Effects of aging and weaning on mRNA expression of leptin and CCK receptors in the calf rumen and abomasum. Domest. Anim. Endocrinol. 22:25-35.

Zened, A., S. Combes, L. Cauquil, J. Mariette, C. Klopp, O. Bouchez, A. Troegeler-Meynadier, and F. Enjalbert. 2013. Microbial ecology of the rumen evaluated by 454 GS FLX pyrosequencing is affected by starch and oil supplementation of diets. FEMS Microbiol. Ecol. 83:504-514. 